\title{
Indeterminacy of a Free Choice: Ontic, Epistemic, or Logical?
}

\author{
Helmut Fink \\ University of Erlangen-Nürnberg
}

\section{THE PROBLEM OF ALTERNATE POSSIBILITIES IN THE DEBATE ON FREE WILL}

Cognitive events are characterized by a notorious dichotomy of their
possible modes of description. On the one hand, an introspective description can be given by a succession of mental states of a person. Mental states refer to conscious perceptions, thoughts and feelings the person has. These states cannot by themselves be "seen" from the outside. Strictly speaking, they can only be given meaning from a first-person perspective: It is only me who knows what it is like to be myself. On the other hand, an external description of cognitive events can be given by the dynamics of the neural states of the person's brain. Neural states are states of matter. They can be measured from the outside and get their meaning from a third-person perspective.

The question how exactly these two modes of description relate is a very far-reaching one. In philosophical tradition, this question lies in the center of what has become well-known as the mind-body-problem. In modern neuroscience, the search for neural correlates of mental states has proven very fruitful. In cognitive science as in daily life, comparing mind talk with body experience is the ultimate reason for ascribing mental states, consciousness and the like to other living beings. In any case, it is a basic building block of every naturalistic world-view (and even some moderate anti-naturalistic ones) that mental states are always accompanied by a material basis.

In this article, we are only concerned with a special case of cognitive events, albeit a very important one: the case of a free choice of an agent. 
Driven by experimental results on the temporal order of neural versus mental states (Libet 2004) and on the deceivability of the feeling of control (Wegner 2002), the debate on free will has been intensified among scholars in science and philosophy in recent years and at the same time spread out in popular journals and the media. The neural correlates of mental states obey the laws of physics. Hence: Do we have free will, and if so, in which sense? Or do we have to accept, at least at a scientific level, that all we want and do is actually determined by the matter of our neurons-and not by us? Is free will an illusion, even though a useful one?

The relevance of this debate exists without doubt: Our view on acting persons in contrast to moving bodies involves a notion of free will. Our customary concept of moral responsibility depends on the possibility of a free choice. And the traditional criteria for guilt in criminal law hinge upon the freedom of the culprit's choice. One of the (perhaps very few) genuine philosophical contributions to debates like this is the search for a precise reconstruction of concepts that are phenomenologically proven (or appear to be so).

For the concept of free will, three principles or, better, three conditions have been identified. The first one is the condition of authorship: A free decision to act should be attributable to the agent. The person who decides should be, in some sense, the initiator of the decision. Second, a free decision should be intelligible, that is, it should be possible to give reasons and to understand reasons for and against a certain decision. Third, there should have been, in an appropriate sense, alternate possibilities for making the choice. It should have been possible to decide differently, so that counterfactual propositions ("if I had chosen otherwise ...") are meaningful.

As can easily be seen, these three conditions cannot be maintained simultaneously if each of them is interpreted in a strong sense: Assume a strong understanding of authorship. This implies a deterministic relation between cause and effect, because without underlying strong causal lines the attribution of authorship would become uncertain. But then, if determinism applies also to neural dynamics (as it has to), there is no room left for alternate possibilities in a given situation. Conversely, if there is no sufficient cause for the next link in a causal chain, such that alternate 
possibilities show up e.g. in neural dynamics, then this causal gap undermines the attribution of authorship.

Further, if intelligibility is taken for granted, one is committed to the level of reason given by the decider. But deterministic causal lines for the underlying dynamics of his/her brain would certainly lead back to material conditions outside the decider's body and even before his/her birth. This kind of consequence argument highlights a tension between intelligibility and determinism. But the tension between intelligibility and indeterminism is at least as strong: Pure chance is the natural enemy of a definite reason.

As a consequence of these considerations, some or all of the three conditions given above have to be weakened. A strong concept of free choice would be inconsistent (an insight that had been well-known, by the way, to some philosophers long before brain imaging achieved its presentday publicity). Yet, there is no unique recipe how to weaken the conditions for free will. Since the naive expectation can only partially be reconstructed, there may be different consistent concepts of free will, according to different combinations or interpretations of the conditions maintained.

In order to save the intuition of free will (which is more than the freedom to act), we prefer a compatibilistic stance. In this context, compatibilism stands for accepting compatibility of the free will of persons with the determination of all neural processes by natural law. The resulting view on free will is perfectly consistent with naturalism, as has brilliantly been argued for (Beckermann 2005). To this elaborated position, we can add not much more than some explanatory remarks.

First, the key to accept compatibilism is to replace whether a decision is determined by how it is determined. A decision is called free, if the person who made it could judge his/her desires without coercion or compulsion. There is no freedom without deliberation. The freedom of a person's will lies in its coincidence with the person's long-term preferences and rational interests. In a sense, free decisions can be said to be determined by argument and reason. It is this process of deliberation by which a person acquires his/her free will. The resulting free decision is emphatically his/hers. People are the authors of their decisions. But there is no ultimate authorship that could substitute for a decision's causal prehistory. 
Second, the key to avoid methodological confusion is to keep the different modes of description separated. One can either give reasons or study causes, but not both at the same time. To give a reason is to communicate a particular content of a person's conscious thought, while a cause has to be looked for in the behavior of matter. Reasons and causes are elements of two completely different language games: "mind talk" on the one hand and "matter talk" on the other hand. One may object that a good reason is never subjective but always intersubjective. This is true insofar as one may abstract from all individual instantiations of a mental content, and it is true in particular if this content is a reason. But nonetheless reasons, like intentions or aims, are not part of our physical description of the world; causes are.

In a causally closed material world, there is no proper causal role to play for the mind as distinct from the matter. Ontologically, one may choose from a range of positions compatible with this insight, among them e.g. neutral monism or physicalistic epiphenomenalism. We need not be so ambitious as to decide this question here. In any case, mixing up mental states with neural states is an epistemological category mistake. Reasons have no causal power just as causes have no persuasive power. Hence, one should beware of hybrid concepts like "mental causation". Causal links can only be found within the physical mode of description. By virtue of neural correlates of consciousness (or conscious correlates of neural dynamics), it is always possible and often useful to change the mode of description of a cognitive event. But the link between these modes is not a causal one.

For a reason to "determine" a decision, a causal bypass within the physical world is called for: switch from the instantiation of the reason to the underlying neural state, study its causal dynamics, and then switch back to the mental mode of description. If you regard this procedure as irrelevant to your purpose, then you are most likely interested not in causation but in reasoning. For the purpose of reasoning, it is methodologically consistent to stay in the mental mode of description all the time. It is in this mode that a free decision fulfills the condition of intelligibility.

Third, the key to understand authorship is to reconstruct the concept of a person. In addition to the modes of description introduced so far, there are also different levels of description: One can either stick to the most 
elementary building blocks of a complex object (or subject) to be described, or introduce concepts at a more phenomenological level. For example, phenomenological thermodynamics is provided with its own concepts, although the underlying degrees of freedom of the matter under study can be measured and described at the microscopic level (at least in principle). This microscopic description may be regarded more fundamental, but it is not appropriate for every purpose. To be sure, a macroscopic description must not contradict the predictions on the microlevel. But the hierarchical structure of the world we live in suggests using phenomenological concepts on the macro-level without a bad methodological conscience. The concept of a person is on the macro-level. In this concept, aspects of the physical as well as of the mental mode of description are combined: A person consists of his/her body including the brain, and also of the stream of consciousness accompanying its neural dynamics. Persons are highly structured accumulations of matter, together with the perceptions, thoughts and feelings that appear from the correlated internal ("first-person") perspective.

To introduce the personal level of description in this way may not be sufficient in order to define the concept of a person. As was stressed by Frankfurt (1971), persons should be characterized by the structure of their will. But in any case, introducing the personal level is necessary to trace back authorship to the internal control mechanisms of a person. Once this personal level of description is accepted, there is no point anymore in looking for prior and exterior causes explaining the person's free decision. To be sure, the existence of such causes is not denied. However, considering them is not appropriate for the purpose of understanding authorship and is, therefore, stopped by a methodological cut.

Propositions about persons cannot simply be replaced by propositions about some of their parts. Phrases like "the brain decides" instead of "the person decides" give rise to misunderstandings concerning free will, all the more if a deterministic brain dynamics is supposed. Mixing up a whole and its parts in this way amounts to the so-called mereological fallacy. Notably, all our remarks about the concept of a person refer to methodological consistency within a naturalistic approach. They are not meant to suggest that persons are metaphysical entities or somehow excluded from nature's law. In fact, the opposite is true. 
Combined, the conceptual reconstruction of "free will" involves a mental mode and a personal level of description. The concept of free will is of great phenomenological importance. To call a decision "free" helps to distinguish its mechanism from pure chance on the one hand and from coercion or compulsion on the other one. This phenomenological distinction has nothing of an illusion. However, our conceptual reconstruction seems not yet complete. Taken for granted authorship (by persons) and intelligibility (by reasons), what about the condition of alternate possibilities? How can we do justice to the strong intuition that a person's decision has been free only if they could have decided differently?

\section{THREE KINDS OF INDETERMINACY}

If alternate possibilities are to play some role in the description of a decision at all, some element on some stage of the decision process must be, at least in some sense, indeterminate. Conceptually, one can distinguish three different kinds of indeterminacy: ontic, epistemic, and logical. Ontic indeterminacy refers to "things as they really are", while epistemic indeterminacy refers to "things as they appear to us". Epistemology is about our knowledge and its limits. Epistemological propositions about perceptions, thoughts or feelings do not imply ontological claims about the exterior world. Both ontic and epistemic indeterminacy will be discussed in the present section. Logical indeterminacy will be shortly explained at the end of this section but criticized in the subsequent one.

It is reasonable to accept that there is ontic indeterminacy in the world. One of the two major revolutions in $20^{\text {th }}$ century physics has given rise to quantum theory. Compared to classical physics, among the basic characteristics of quantum theory is its power to quantify ontic (objective) indeterminacy, e.g. by Heisenberg's uncertainty relation, and to provide probabilistic predictions for the not-yet-determined values of physical (measurable) quantities. Quantum probabilities do not merely reflect the subjective ignorance of an observer (as classical probabilities do). Leaving aside bizarre extensions of the quantum formalism that are on the run from Occam's razor (like Bohmian mechanics), one can prove mathematically that it is inconsistent to attribute definite values of all physical quantities to 
a quantum system simultaneously. Definite values appear only in a measuring apparatus, where they become visible e.g. as a pointer position.

Can we make use of quantum theory in the area of free decisions? We think the answer to this question is in the negative. It is true that there is a break in the deterministic behavior of matter wherever a quantum effect shows up: If a quantum system is prepared twice in exactly the same quantum state and exactly the same quantity is measured each time, one can nevertheless obtain two different results. So there are alternate possibilities for the behavior of matter even at the most microscopic level of description. But these possibilities are of no use for the concept of free will, as was indicated quite at the beginning of this article: Breaking the chain of causal links inevitably undermines the attribution of authorship. If ontic indeterminacy comes into play on a critical stage of a decision process, then this decision is not free but arbitrary (within the given range of possibilities). A relevant part of the neural dynamics correlated with the process of deliberation is subject to pure chance then. Every quantum process is like a tiny random number generator in the decider's brain. This is a model of diminished responsibility rather than of free will.

Within the interpretation debate on quantum theory, there have been a few attempts to relate measurement outcomes with the consciousness of the observer. But every single outcome is a fact, and the quantum statistics of outcomes is determined by the physical situation. Facts as well as their statistics are part of the material world. They are not (only) in the mind of some observer. Quanta are not qualia-after all.

Conceptually, ontic indeterminacy seems to be irrelevant to reconstructing the essential features of free will. Even worse, randomness comes as a threat to authorship and intelligibility. Hence, pure chance is no chance of free will.

Fortunately, quantum effects seem to be irrelevant to neural behavior also empirically. The physiological processes relevant to perceptions, thoughts and feelings can be described in classical concepts, at least according to the vast majority of neuroscientists. There is no convincing neurophysiological evidence of quantum effects in brain dynamics so far. This observation completes our discussion of ontic indeterminacy. In order to avoid mixing up the different kinds of indeterminacy, we will assume a 
classical, deterministic universe while considering epistemic and logical indeterminacy.

Epistemic indeterminacy refers to a limited state of knowledge either of some external observer, i.e. from a third-person perspective, or of the decider him-/herself, i.e. from a first-person perspective. Let us first consider the "view from the outside". If an observer wants to predict a free decision of someone else, he/she may study this person's usual behavior and look for determinants of the decision in the causal prehistory and in the environment of the decider. In view of physical law, the best the observer can do is to study the decider's neural dynamics.

Under natural circumstances, the observer's prediction will not always be successful. He/She may have overlooked one or another causal influence in the prehistory of the decision. Or his/her theoretical model of the human brain may have been too simple for a physical system as complex as this. Perhaps his/her computer power has not been sufficient. Concerning the concept of free will, none of these obstacles to a prediction of a person's decision appears as fundamental. Causal determinants can be investigated, theoretical models can be improved, computer power can be increased. In this way, alternate possibilities for the decision under study can be successively excluded until, in the limit of infinite ressources, the observer's prediction becomes unique. But one would not say that a free decision becomes less free just by sharpening an observer's prediction. Hence the condition of alternate possibilities in the debate on free will cannot convincingly be fulfilled by subjective ignorance of an observer.

We add three remarks. First, the knowledge about all the relevant causal influences is hard to achieve. From a practical point of view, unique predictions of free decisions will remain impossible for a long time. But our main concern here is conceptual rigor, not practical prospects. Therefore we refer to idealizations where it seems helpful.

Second, one may argue that the neural processes accompanying the deliberation of a free decider are perhaps algorithmically irreducible. If this were true, no observer of a decision process could exclude alternate possibilities by computational means until the process is finished. In other words, the neural process was the shortest possible solution to the prediction problem. In view of the imperfection of almost all human capacities we regard this assumption as not very plausible. It may be 
conceivable as an exceptional case. But we are mainly interested in the regular case.

Third, one could prefer describing the decision situation on a macroscopic level rather than on a microscopic one. Even if a unique prediction is regarded possible, it may seem appropriate to refrain from specifying the situation completely. Situations in daily life are usually not being described by specifying the initial conditions of every potentially relevant degree of freedom. Rather, parts of the environment, including one's fellow human beings and their behavior, are treated like a variable. In such a partially specified description, a person's decision is still open to be influenced. Alternate possibilities appear.

Nevertheless, in a deterministic world, the person can decide differently only if at least one of the initial conditions is different. Hence the macroscopic level of description outlined here corresponds to a certain probability distribution of initial values in a microscopic description. In analogy with the methodological cut introduced for reconstructing personal authorship, it is an at first sight plausible approach to underpin the intuition of alternate possibilities by subsuming different deterministic microhistories under a common description on a personal level.

However, coarse-graining the description in this way is by no means a necessary consequence of introducing the personal level: authorship can perfectly be reconstructed by reference to the internal control mechanisms of a person even if all initial conditions are exactly specified. In any case, it is legitimate to consider such a fine-grained description for theoretical reasons. From this perspective, however, alternate possibilities do appear as an artefact of coarse-grained descriptions. Thus, one might call alternate possibilities an epistemological illusion, if epistemic indeterminacy from a third-person perspective were the only approach to reconstruct their meaning.

Independently of the level of description chosen, and notwithstanding the three remarks just listed, we think that "unpredictable" is a very poor reconstruction of "free". As far as the "view from the outside" is concerned, we conclude that there is no need to identify alternate possibilities at all. In this respect we agree with Frankfurt's influential analysis (Frankfurt 1969), in which he disentangled moral responsibility 
from the principle of alternate possibilities. But still, the "view from the inside" remains to be considered.

By epistemic indeterminacy from the first-person perspective we mean the ignorance of the decider about how he/she will decide. This ignorance seems to be necessary until the decision is really made. Deliberation is essential to freedom, as we argued for in section 1. But the relevant deliberation that precedes a free decision is of a particular kind: arguments for and against different possibilities have to be weighed. Without alternate possibilities, there is no decision to make. If the decider already knows the result of his/her deliberation, one would look for the moment of decision in the past and not in the future.

Thus, this lack of prior knowledge is a convincing candidate for reconstructing the origin of the robust intuition of alternate possibilities in the debate on free will. We stress that predictability is assumed to be limited only from the internal perspective now. For an external observer (with perfect physical knowledge), every neural state of the decider, and hence also the result of the decision process, may be predictable. Decoding the decider's individual reasons from his/her neural states would be a discouragingly difficult task. We repeat that reasons have to be gained by abstraction from certain mental states, which is a different epistemological category from neural states. But by virtue of mental correlates of neural states, hypothetical predictions can be made. These can be tested by comparison with the decider's comments and actions. As a result, the external observer may one day be able to give a unique, and even intelligible, prediction of the decider's next free choice.

Nevertheless, the decider refers to alternatives that he/she regards, and has to regard, as equally possible (though, perhaps, not equally probable) during his/her deliberation process. This epistemic indeterminacy from the first-person perspective seems to be constitutive for the freedom of a person's will. The resulting reconstruction of free will is perfectly compatible with physical determinism. Even if the decider knows that he/she lives in a deterministic world, he/she is still confronted with subjective alternate possibilities. We remark that Walde (2006) has most recently referred to this condition for free will under the notion of epistemic openness of the future ("Epistemische Offenheit der Zukunft"). 
Now, one may ask a far-reaching question: What happens if the decider him-/herself is going to compute the result of his/her own future decision on the basis of the deterministic dynamics of his/her neural states? Or, what amounts to the same, if an external observer communicates a unique prediction of the result before the decider's deliberation process is finished? Certainly, the presence of this information is a major stumbling block for the consideration of equally possible alternatives. Does the decider cease to be free then? Is this scenario consistent at all?

According to Popper's classical essay (Popper 1950), a deterministic system can neither predict its own future state nor receive such a prediction from another system without threatening the validity of the prediction by this very act. Hence one might think that a decider is necessarily in a state of ignorance in comparison with his/her observers.

Strengthening Popper's conclusion, Donald M. MacKay has developed the position of logical indeterminacy (MacKay 1960; 1967). According to this position, there is a relativity between the decider's and the observer's description, which is rooted in their distinct roles. The two descriptions differ from each other, but are uniquely related to each other. According to MacKay, neither of them can be claimed to be objectively true. Therefore the decider has no ignorance, no "lack" of knowledge, and hence the attribute "logical" instead of "epistemic".

Logical indeterminacy explicitly presupposes a deterministic universe. If the position of logical indeterminacy is well-founded, then freedom of the will is built into the decider's perspective with logical necessity, and can thus be deduced (and must be recognized) by every rational observer. However, we have severe doubts about the well-foundedness of this logical indeterminacy of a free choice.

\section{CRITIQUE OF LOGICAL INDETERMINACY}

Doubts are possible already with respect to Popper's conclusions. Popper claimed to have discovered a fundamental epistemic indeterminism pertaining to quantum and classical systems alike. He considered classical mechanical calculating and predicting machines, so-called predictors, which are thought of as realized in the physical world. Hence, unlike the Laplacean demon, predictors have finite resources and the task to predict 
the behavior of finite systems with a specified degree of precision. Popper argued for the thesis that there are prediction tasks which no predictor can perform. In particular, no predictor can fully predict its own future states.

This kind of fundamental unpredictability, if it exists, leads to the consequence that a predictor cannot compute its own disturbing influence on other systems with which it interacts. Hence, this influence should be kept negligibly small. But this is impossible if the other system happens to be itself a predictor that is concerned with the first predictor, because predictors need to amplify weak influences of their object systems. Therefore, a successful predictor of predictors would have to remain outside the "society of predictors".

The essential point is Popper's claim that no predictor can predict its own future states. We think his reasoning for this thesis is worth reconsidering. Parts of it appear to us more confusing than convincing. The intuition behind this reasoning seems to rest exclusively on the consideration of a succession of preliminary predictions, of which each represents the effects of the preceding one. But could not the problem of making a prediction whose effects shall also be predicted be treated in a self-consistent way?

In classical mechanics, a measured system may be disturbed by the act of measurement. But unlike in quantum mechanics, such a disturbance may be calculated exactly in every individual case. What appears as disturbance is nothing but a particular interaction between measurement apparatus and measured system. This interaction obeys the same deterministic laws of nature as interactions within the measured system do. There is no reason why its effects should not be calculable and predictable.

The only additional difficulty in Popper's situation is the structure of self-consistency: what is to be predicted, depends itself on the prediction. But, supposing a sufficiently powerful algorithm, equations representing such a structure may be recursively solved despite Popper's no-go claim. This is at least our, of course fallible, intuition about these matters.

MacKay's reasoning starts from the essential contrast between communicating an observer's prediction to an observed decider and shielding the decider from any influence that could invalidate the prediction. If the prediction is communicated, processing the observer's message affects the decider's neural dynamics. According to MacKay, it 
can never be excluded that this invalidates the prediction. Similar to Popper, MacKay is concerned only with predictions made on an empirical basis that is disturbed if the prediction is communicated to the decider. As above, we feel entitled to consider predictions that remain true if communicated, because they anticipate and include these effects already.

MacKay concludes that the observer's prediction must not be claimed to be objectively true, that is, true for everyone who knows of it and probably wants to test it, because it cannot become true for the decider. For him/her, all such predictions are logically indeterminate until he/she makes his/her choice. To be sure, external observers ("silent onlookers") may test their predictions and find it confirmed. But the role of the decider is, according to MacKay, logically different.

Several critical comments have appeared in the literature against this concept of logical indeterminacy. MacKay replied to all of them and gave the impression that he succeeded in defending his view (MacKay 1971, 73). As an attack to MacKay's "logical relativism", Watkins (1971) is still worth reading. Watkins - as a logical non-relativist-reconstructs the status of the predictions about a decider quite convincingly by use of ceteris paribus-clauses.

What shall we make out of it? If logical indeterminacy is refused, we are back to epistemic indeterminacy from the first-person perspective. If there are predictions about a decider that can be claimed to be true objectively, then the decider's lack of prior knowledge deserves to be called subjective ignorance. But then, what does it mean to remove this ignorance?

As a consequence of our remarks made above about self-consistent problems, we have to postulate that there may be trajectories in the space of neural states of a decider that represent a behavior consistent with calculated prior knowledge, or with communicated true predictions. This trajectory in the physical description corresponds to a stream of consciousness in the mental mode of description. We admit that the following consideration is highly speculative and far from all practical purposes. But we think it belongs to a complete conceptual reconstruction of a free choice and hence of free will. 


\section{TEMPORARY DELIBERATION AS A MINIMAL CONDITION FOR FREE CHOICE}

To know a true prediction about one's own future decisions looks like a contradiction in itself. It is tempting to regard "making the choice" and "knowing the result of the choice" as one and the same thing. But there is a subtle distinction that helps to avoid the contradiction alluded to above. "To know (in principle)" is not the same as "to think now (consciously) of". If someone knows something, he/she can tell you about it when being asked. This does not imply, of course, that the contents of this knowledge is permanently in his/her mind. If it happens to be in mind, we call this an instantiation of the person's knowledge. Consciousness is extremely selective, and only a few thoughts "come to our mind" in a given period of time. Free decisions are rooted in deliberation processes, as we saw in section 1. Judging one's desires is a mental process that needs some time. Only by this process can a decider acquire a decision and learn to look at it as his/hers. It is true that alternate possibilities are in the mind's eye of the decider as long as he/she thinks about them. But this process of deliberation may have a break. In such a break, absolutely different contents may come to the decider's mind. Later on, he/she continues with deliberation.

Now, if a decider gets knowledge about the result of his/her decision, then he/she certainly stops deliberating while this knowledge is instantiated in his/her mind. But the task of conscious deliberation is not instantly fulfilled only because its result is known. At a later moment, the decider may continue deliberating. There is, at least, no logical contradiction in this succession of mental states. Admittedly, from a psychological perspective, this patchwork stream of consciousness may seem a little schizophrenic. But we are analyzing an extreme situation, so we need not be surprised by an extreme conclusion.

Summing up, the intuition of alternate possibilities is a crucial ingredient to the project of reconstructing the concept of free will. In order to make this concept consistent and fruitful, the condition of alternate possibilities has to be weakened. The relevant alternate possibilities are grounded in epistemic indeterminacy rather than ontic or logical indeterminacy. 
Ontic indeterminacy implies unpredictability of individual future events. Realized in quantum effects, this sort of indeterminacy is too strong to be helpful for a free will. Logical indeterminacy does, as far as we can see, not exist at all. Epistemic indeterminacy from a third-person perspective, ubiquitous as it may be, misses the point of free will. To be unpredictable from outside is not to be free. In addition, predictability may be increased without loss of freedom (hopefully).

Epistemic indeterminacy from a first-person perspective, however, remains constitutive for free decisions, even if it has to be restricted to certain periods of deliberation. But this restriction is a burden only on deciders who prefer predicting their decisions instead of making them.*

\section{REFERENCES:}

Beckermann, A. 2005: "Free will in a natural order of the world." In:

Nimtz, C. and Beckermann, A. (eds.) Philosophy-ScienceScientific Philosophy. Paderborn: Mentis.

Frankfurt, H. G. 1969: "Alternate possibilities and moral responsibility." Journal of Philosophy 66, 829-839.

Frankfurt, H. G. 1971: "Freedom of the will and the concept of a person." Journal of Philosophy 68, 5-20.

Libet, B. 2004: Mind Time. Harvard: Harvard University Press.

MacKay, D. M. 1960: "On the logical indeterminacy of a free choice." Mind 69, 31-40.

MacKay, D. M. 1967: Freedom of Action in a Mechanistic Universe. Cambridge: Cambridge University Press.

MacKay, D. M. 1971: "Choice in a mechanistic universe: a reply to some critics." The British Journal for the Philosophy of Science 22, 275285.

MacKay, D. M. 1973: "The logical indeterminateness of human choices." The British Journal for the Philosophy of Science 24, 405-408.

I am indebited to Georg Gasser for his editorial patience and to Anja Göritz for her critical thinking. 
Popper, K. R. 1950: "Indeterminism in quantum physics and in classical physics." The British Journal for the Philosophy of Science 1, 117133, 173-195.

Walde, B. 2006: Willensfreiheit und Hirnforschung. Das Freiheitsmodell des epistemischen Libertarismus. Paderborn: Mentis.

Watkins, J. W. N. 1971: "Freedom and predictability: an amendment to MacKay." The British Journal for the Philosophy of Science 22, 263275.

Wegner, D. M. 2002: The Illusion of Conscious Will. Cambridge, MA: MIT-Press. 